\title{
Preliminary Variations in Physiological Modules When sub1A QTL Is under Soil-Moisture Deficit Stress
}

\author{
Indraneel Saha1, Arnab Kumar De1, Arijit Ghosh1, Bipul Sarkar1, Narottam Dey², \\ Malay Kumar Adak ${ }^{*}$
}

${ }^{1}$ Department of Botany, University of Kalyani, Kalyani, India

${ }^{2}$ Department of Biotechnology, Visva-Bharati University, Santiniketan, India

Email: ^mkadak09@gmail.com

How to cite this paper: Saha, I., De, A.K., Ghosh, A., Sarkar, B., Dey, N. and Adak, M.K. (2018) Preliminary Variations in Physiological Modules When sub1 $A$ QTL Is under Soil-Moisture Deficit Stress. American Journal of Plant Sciences, 9, 732-744. https://doi.org/10.4236/ajps.2018.94058

Received: January 24, 2017

Accepted: March 16, 2018

Published: March 19, 2018

Copyright $\odot 2018$ by authors and Scientific Research Publishing Inc. This work is licensed under the Creative Commons Attribution International License (CC BY 4.0).

http://creativecommons.org/licenses/by/4.0/

\begin{abstract}
The present investigation embodies with some distinct physiological responses of rice seedlings bearing $s u b 1 A$ allele when cultured under soil moisture deficit condition. In order to estimate the extent of variation of $s u b 1 A$ allele in cv. Swarna Sub1, few specific responses were recorded. Ongoing days (2, 4 and 8$)$ of water stress, rice seedlings were mostly concerned with regulation of root growth through $1.24 \%, 4.10 \%$ and $8.96 \%$ as compared to control. When relative growth rate (RGR) of the biomass was considered, it recorded a significant down regulation maximum by $27.27 \%$ over non-stressed plants. The light absorbing capacity of foliages in terms of leaf mass ratio (15.62\%) and specific leaf weight (8.45\%) was also curtailed under stress contiguously with total dry matter accumulation. For light harnessing mechanism, the contributing pigments were most significantly $(\mathrm{p} \leq 0.05)$ affected with maximum variation for chlorophyll (31.29\%), lycopene (79.31\%) and carotenoids (55.31\%) over control. NAR was strikingly differed by $32.72 \%$ under water stress in average. To support the water stress related depletion of moisture tension, plants recorded RWC with a ranges of $6.62 \%$ to $21.57 \%$ under stress than control. In balance of cellular dehydration rice seedlings were recorded $6.72 \%$ loss of proline, but up-regulated the release of free amino acids by 1.35 fold. In expression of generic potential for subl $A$ allele, the growth inhibition even under water deficit was evident from carbohydrate depletion by $10.93 \%$, $11.42 \%$ and $24.35 \%$ through days of treatments. This was supported by depletion of apoplastic and cellular invertase activities by $90.90 \%$ and $72.91 \%$. The possibility for $\operatorname{subl} A$ in tolerance of deficit water status in rice culture has been discussed in more details.
\end{abstract}




\section{Keywords}

$s u b 1 A$, Rice, Water Deficit, Growth, Metabolism

\section{Introduction}

Submergence or flooding stress on rice culture is quite consistent in detrimental effects with depth and duration in the field. The analysis of submergence related reactions on rice land-races is focused with two domains: the inundation of hypoxic/anoxic effects of seedlings and the concomitant oxidative stress at post submergence period with receding of water level. This occurs due to exposure to high oxygen tension when the seedlings are released from water regimes [1]. Therefore, land races are escaped with submergence by the physiological responses related to oxidative damages of bio-molecules. However, in rainy seasons the delayed or infrequent monsoon may often induce some inadequate moisture level to the low land condition. Rice cultures particularly, with semi-tall indica varieties under this situation may often meet the depleted moisture level around rhizosphere. This often creates some sort of moisture stress even in non-submergence condition for the low land rice [2]. This is more experienced with the loss of chlorophyll in the foliages as well as earlier permanent wilting per cent under such soil below saturation point of moisture. On identification of submergence tolerant lines either following escape or quiescence strategy has been functional with other allied cellular responses. For the elongation of internodes and leaf sheaths to accomplish the escape of submergence stress, the semi-tall land races are the best choice, however, facing with lodging and low yield potential [3]. On the other hand the growth suppression factors through ethylene mediated down regulation are the key to quiescence strategy. This is characterised with arrest of carbohydrate utilization from storage tissues to ensure suppressed growth under water [4]. With this, the dwarf high yielding varieties are obtained by exercising the quiescence for survival than escape strategy under complete submergence. In true sense quiescent lines with their characteristic cellular responses are more prone to oxidative stress perception following downstream response pathways. Still, the probability of quiescence traits in rice cultivars for their certain exposure with high moisture tension in soil during water deficit may open another line of investigation. It is well admitted that under submergence due to low or depleted dissolved oxygen around rhizosphere, the plants are often experienced with water stress. This is mostly due to inadequate root-hydraulic conductance [5]. Therefore, the information and understanding regarding the fates of rice seedlings when exposed to earlier drought condition are also relevant to justify. With this background we have evaluated a well known submergence tolerant line, cv. Swarna Sub1 possessing the $\operatorname{sub} 1 A$ quantitative trait loci (QTL) under simulated condition of soil moisture depletion. The present work in brief describes and discusses a few drought responses 
which might support the nature of reaction of $\operatorname{subl} A$ when interacting with soil moisture deficit.

\section{Materials and Methods}

\subsection{Development of Seedlings, Water Stress and Sample Collection}

The entire experiment was conducted in experimental garden and laboratory of Plant Physiology in the department of Botany, University of Kalyani, Kalyani $\left(22^{\circ} 58^{\prime} 30^{\prime \prime} \mathrm{N}, 88^{\circ} 26^{\prime} 04^{\prime \prime} \mathrm{E}\right)$, West Bengal, India. Seeds of rice (Oryza sativa L.) cultivar were used for this study as experimental material. The typical submergence tolerant line cv. Swarna Sub1 was collected from farmers' field. Sterilised seeds (by immersion in $1 \%$ sodium hypochloride solution following repeated rinse) were germinated on moist filter paper for four days at $28^{\circ} \mathrm{C} \pm 2{ }^{\circ} \mathrm{C}$ in a growth chamber. Sprouted seeds were placed on a wire net covered with moist cotton in a $1 / 4^{\text {th }}$ strength of medium [6]. Seven days old seedlings (2 to 3 leaf stage) with adequate developed roots were transferred in earthen wire pots $(20 \mathrm{~cm}$ dia) filled with alluvial clay loam of lower Gangetic plain ( $\mathrm{pH}$ 6.8) soil. The experiment was conducted through induced water stress by withholding the irrigation continued for 10 days. Seedlings @ of 5 to 6 per hill were transplanted by spacing $20 \mathrm{~cm}$ apart and $10 \mathrm{~cm}$ between hills. Fertilizers were subjected to soil @ 20, 15, $15 \mathrm{~kg}$ per hectare in the form of $\mathrm{N}: \mathrm{P}_{2} \mathrm{O}_{5}: \mathrm{K}_{2} \mathrm{O}$. The plant was kept for 8 days under the condition of appropriate light and dark cycle (16L:8D), light intensity of 900 $1200 \mu \mathrm{Em}^{-2} \cdot \mathrm{S}^{-2}$ of photon flux density, $85 \%$ of relative humidity, $30^{\circ} \mathrm{C}-35^{\circ} \mathrm{C}$ of temperature. On preliminary observation the mortality of the plants were recorded maximum within 10 days. Thus, sampling at intervals of 2, 4 and 8 days were done to examine plants perceptions and reactions under water stress. A control set was made where watering was throughout the period. The plants were replicated thrice in randomised block design in independent experiment for each period of sampling (2, 4 and 8 days). All the sets were placed in natural open air condition. After treatment the samples from each replication with 5 plants were transferred to storage by freezing in liquid nitrogen at $-80^{\circ} \mathrm{C}$ for chemical analysis.

\subsection{Determination of Growth Parameters}

The plants were collected from each treatment at the end of their respective duration. The samples for roots and shoots were dried in hot air oven at $80^{\circ} \mathrm{C}$ till constant weight. The growth performance was evaluated by different parameters including net assimilation rate (NAR), leaf mass ratio (LMR), relative growth rate (RGR), specific leaf weight (SLW) etc. by computing dry weight basis [7]. The length of primary roots was measured by a centimetre scale properly.

\subsection{Determination of Pigments Content}

The freshly harvested leaf samples were thoroughly washed and profiles of dif- 
ferent pigments were analysed by photometry (Cecil, CE7200) using appropriate solvents. For chlorophyll, $100 \mathrm{mg}$ of leaf tissue was extracted in $80 \%$ acetone (MB grade, SRL) following purification of other organic matters [8]. The solvation of lycopene and carotenoids was done with petroleum ether following separation in a column. The amount of lycopene and carotenoids were estimated by reading the absorbance at $503 \mathrm{~nm}$ and $450 \mathrm{~nm}$ respectively with petroleum ether as blank [9]. The amount of pigment was expressed on fresh weight basis.

\subsection{Determination of Stress Metabolites}

As an index of osmotic stress plants were evaluated on the basis of compatible solutes: proline and free amino acids from tissue. $100 \mathrm{mg}$ fresh tissue was crushed in liquid nitrogen and thoroughly homogenised with $3 \%$ aqueous sulphosalicylic acid. The aqueous phase recovered through centrifugation was reacted with acid ninhydrin solution ( $1.25 \mathrm{~g}$ ninhydrin in $30 \mathrm{ml}$ of glacial acetic acid and $20 \mathrm{ml}$ of $6 \mathrm{M}$ phosphoric acid) in warm condition. On boiling the reaction was completed and transferred to ice bath to collect the upper phase with saturated toluene. From the clear solution the amount of proline was determined by reading the absorbance at $520 \mathrm{~nm}$ as suggested by Bates et al. (1973) [10]. L-proline was used for calibration within range of $0.02-0.1 \mu \mathrm{M}$ per $\mathrm{ml}$. The free amino acids content from hydrolysed sample was determined according to Misra et al. (1975) [11] in 80\% aqueous ethanol. The concentrate fraction of amino acids was incubated in a reaction mixture of saturated ninhydrin solution $(20 \mathrm{~g}$ of ninhydrin in $500 \mathrm{ml}$ of methyl cellosolve). The diluents used as equal volume of water and $n$-propanol. The intensity was measured at $570 \mathrm{~nm}$ and expressed on fresh weight basis with standard as leucine.

\subsection{Determination of Water Status in Stressed Plants}

The hydration status of plants under induced water stress was evaluated by relative water content according to Barrs and Weatherley (1962) [12]. RWC was measured by the equal number of fresh leaves from each treatment and taken fresh weight (FW) immediately. Those were hydrated in distilled water fully submerged for 12 hours to the turgidity weight (TW). On transfer at $80^{\circ} \mathrm{C}$ the constant weight was recorded. RWC was computed with the derivation (FW $\mathrm{DW}) /(\mathrm{TW}-\mathrm{DW}) \times 100$.

\subsection{Determination of Carbohydrate Metabolism}

Total carbohydrate is done according to Yoshida et al. (1976) [13]. $100 \mathrm{mg}$ of dry samples from both controls and treatments were hydrolysed for three hours with $5 \mathrm{ml}$ of $2.5 \mathrm{~N}-\mathrm{HCl}$. Thereafter, they were neutralised with solid sodium carbonate until the effervescence ceases. The supernatants were collected by centrifugation $10,000 \times \mathrm{g}$ for $15 \mathrm{~min}, 4^{\circ} \mathrm{C}$ and saved. The assay mixture consisted of $1 \mathrm{ml}$ supernatant added with $4 \mathrm{ml}$ of anthrone reagent $(200 \mathrm{mg}$ anthrone in $100 \mathrm{ml}$ of ice cold $95 \% \mathrm{H}_{2} \mathrm{SO}_{4}$ ) under cold condition. The green colour developed on boil- 
ing water bath was read at $630 \mathrm{~nm}$ by using a spectrophotometer. Finally, the estimation of total carbohydrate was determined by the help of standard curve of glucose and expressed dry weight basis.

Soluble fraction of invertase from leaf extract (100 mM Tris- $\mathrm{HCl}, \mathrm{pH} 4.8 ; 10$ $\mathrm{mM} \mathrm{MgCl} 2,0.1 \%$ BSA, $0.1 \mathrm{mM}$ DTT, $0.1 \mathrm{mM}$ PMSF) was partially purified from $80 \%$ ammonium sulphate cut. The fraction of purified protein was concentrated and assayed with Bradford reagent taking standard of BSA [14]. In assay mixture of acid invertase activity with $100 \mu \mathrm{g}$ was reacted with $200 \mathrm{mM}$ sucrose in 20 $\mathrm{mM}$ sodium citrate buffer ( $\mathrm{pH} 4.8$ ), incubated at $37^{\circ} \mathrm{C}$ for $90 \mathrm{~min}$ [15]. Then the reaction was stopped with alkaline solution on boiling water bath for $10 \mathrm{~min}$ and the absorbance was read at $510 \mathrm{~nm}$. The wall bound fraction was recovered from pooled residue of proteins from leaf extract. The pellet was repeatedly washed with $5 \mathrm{M} \mathrm{NaCl}$ buffer until the Bradford gives any colour. The pellet was dissolved in $150 \mu \mathrm{l}$ of sodium acetate on gentle shaking followed by $10,000 \times \mathrm{g}$ at $4^{\circ} \mathrm{C}$ for $15 \mathrm{~min}$. The assay mixture of $1 \mathrm{ml}$ consisted of $200 \mathrm{mM}$ sucrose in 20 $\mathrm{mM}$ sodium citrate buffer ( $\mathrm{pH} 3.8$ ). The activity was determined in a similar manner as described for soluble fraction.

\subsection{Statistical Analysis}

Data herein were computed by analysis of variance coupled with student's $t$-test (SPSS software IBM, USA). Significance of variance was granted at $p \leq 0.05$.

\section{Results}

\subsection{Variations of Growth Attributes of $s u b 1 A$ under Varying Durations of Water Stress}

From the course of experimental period the different physiological responses of subl $A$ QTL were significantly varied encountering the osmotic/water deficit stress. Water stress through varying durations $(2,4$, and 8 days) was more crucial at the maximum period as recorded from maximum subdued behaviour for almost the physiological attributes. Thus, on initial changes, the root pattern in its length was almost unaffected through the days. Still, at 8 days of stress this was recorded $8.96 \%$ less than control but non-significant ( $\mathrm{p} \leq 0.05$ ) (Figure $1(\mathrm{a})$ ). Unlike root behaviour, changes of chlorophyll with other two pigments (lycopene and carotenoids) were marked reactions for $\operatorname{subl} A$ against water stress. Regardless of duration, chlorophyll, lycopene and carotenoids content were significantly down regulated in a linear manner, still, maximised at 8 days. The variations were significant $(\mathrm{p} \leq 0.05)$ that differed from control and recorded with the value of $31.29 \%, 79.31 \%$ and $55.31 \%$ for chlorophyll, lycopene and carotenoids respectively (Figures $1(\mathrm{~b})-(\mathrm{d})$ ).

\subsection{Variations of Photosynthetic Related Characters under Water Stress}

Therefore, the overall assimilated carbon budget is necessary to evaluate as the plant proceeds through different durations of stress. In the present experiment, 


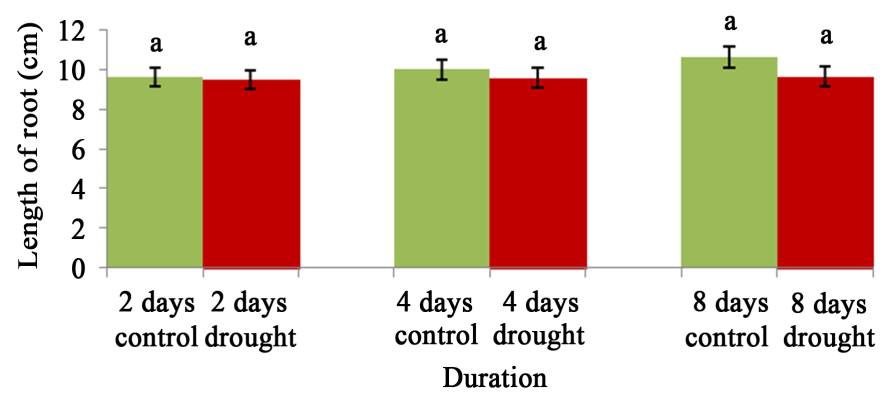

(a)

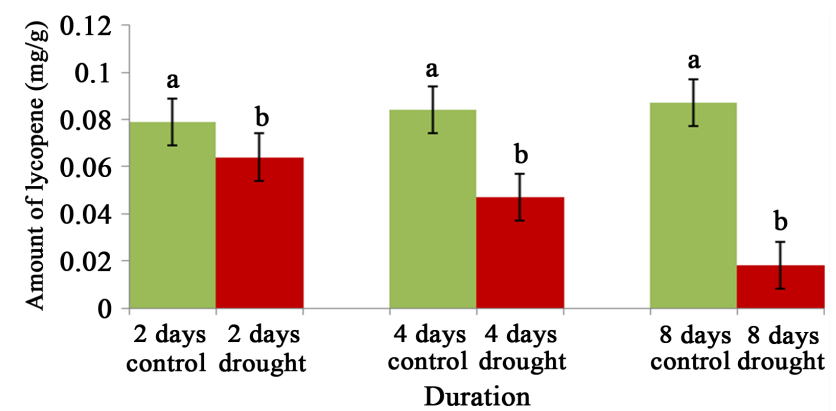

(c)

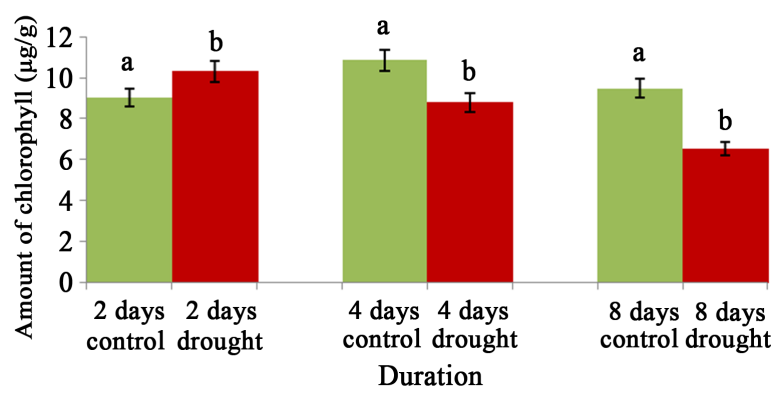

(b)

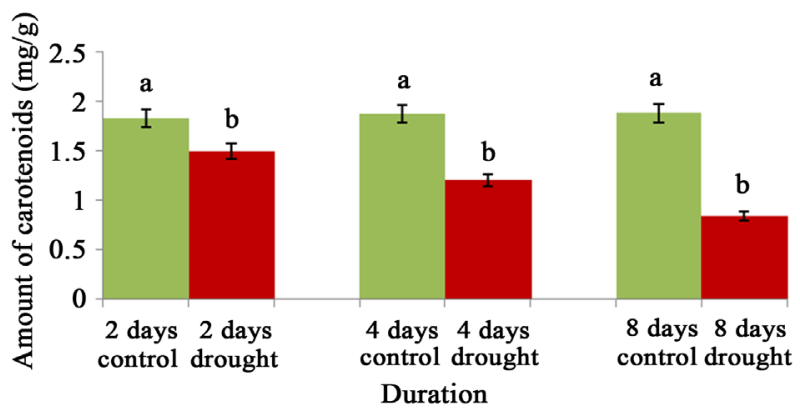

(d)

Figure 1. Changes of root growth (a), chlorophyll (b), lycopene (c), and carotenoids (d) content of cv. Swarna Sub1 through varying days under control and water stress. Data presented in bars with $\pm S E(n=3)$ from independent experimental sets at significance level $\mathrm{p} \leq 0.05$ (by student's $t$-test). Different letters on bar represent significant difference.

$\operatorname{subl} A$ is characterised preliminary with its behaviour of carbohydrate metabolism under water stress through different days. The suppression of growth through regulated photosynthetic carbon assimilation is recorded by a decreasing order of NAR $(34.92 \%, 27.18 \%$ and $32.72 \%)$ against control at different durations (Figure 2(a)). This may also be corroborated with the ratio of leaf to shoot mass (LMR) in an equivalent trend through $8.92 \%, 8.62 \%$ and $15.62 \%$ curtailed under water stress (Figure 2(b)). The conformity of $\operatorname{sub} 1 A$ allele under submergence may be also extrapolated under water stress. This is through total growth with its relative rate (RGR) that is noticed with a significant impairment in the present experiment. Thus, plants have to compromise with its suppressed RGR through the variation of $26.59 \%, 24.63 \%$ and $27.27 \%$ under same (Figure $2(c))$. In concomitant manner the value of LMR and SLW were also the distinguishing features for the impact of water stress on $\operatorname{sub} 1 A$. The ranges for LMR and SLW were appeared to be most sustained and non significant $(\mathrm{p} \leq 0.05)$ in changes of days (Figure 2(b) and Figure 2(d)). Still, at maximum duration of stress the values of LMR and SLW were recorded were $15.62 \%$ and $8.45 \%$ decrease as compared to control.

\subsection{Response of $s u b 1 A$ through Osmotic Characters under Water Stress}

As a consequence of water stress plant tissues must be dehydrated as a mark of plant response. In the present case cv. Swarna Sub1 experienced as usual the decline of osmotic potential, as measured with RWC. The fall in RWC was consistent 


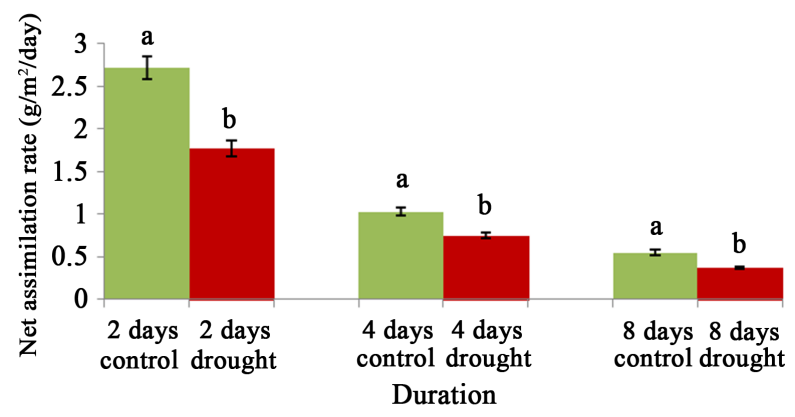

(a)

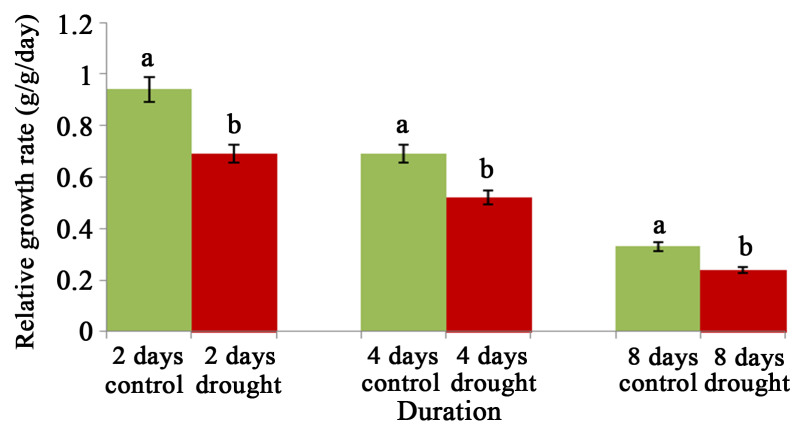

(c)

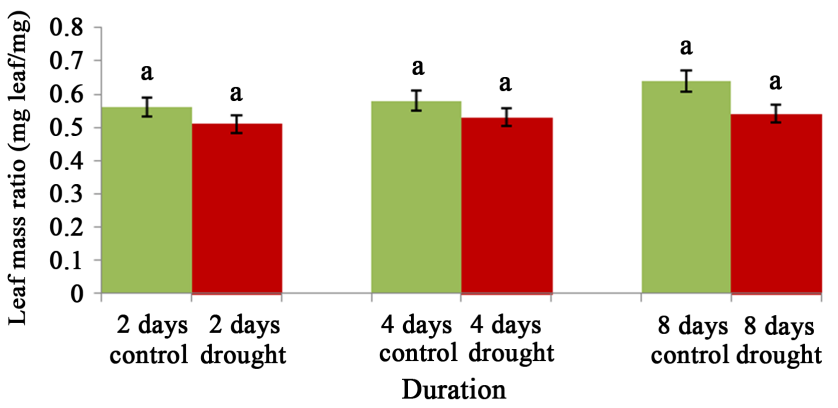

(b)

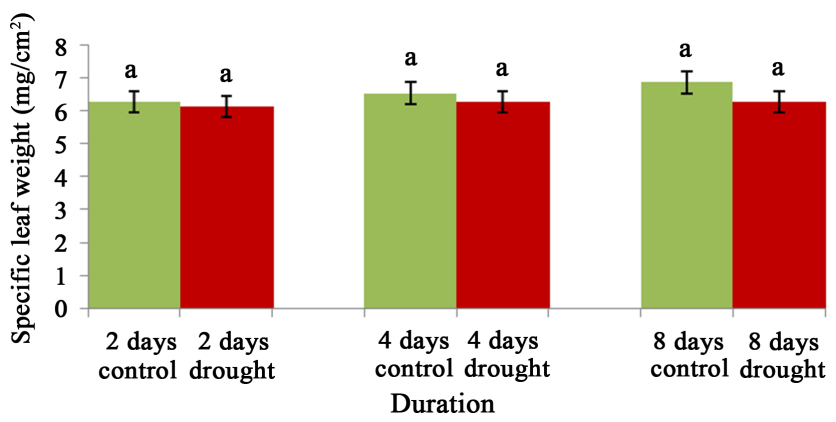

(d)

Figure 2. Changes of photosynthetic rate (a), leaf mass ratio (b), relative growth rate (c) and specific leaf weight (d) of cv. Swarna Sub1 through varying days under control and water stress. Data presented in bars with $\pm \mathrm{SE}(\mathrm{n}=3)$ from independent experimental sets at significance level $\mathrm{p} \leq 0.05$ (by student's $t$-test). Different letters on bar represent significant difference.

in manner and it recorded $6.62 \%, 15.42 \%$ and $21.57 \%$ loss through the days of stress (Figure 3(a)). As expected, plants could have been developed the tolerance strategy with its genetic plasticity and that reflected in noticeable phenomenon with compatible solutes. Proline, the osmoticum is behaved also as a reliable index but not in the cv. Swarna Sub1 variety since a decline of concentration was the feature. The activity of proline had the minimum values under stress at 8 days so recorded over control by $6.72 \%$ less (Figure 3(b)). On the contrary, the release of free amino acids was set as most promising to suppose the osmoticum for this cultivar. The free amino acids level had the up regulation with value of 1.35 fold over control (Figure 3(c)). The opposing trend of two bio-molecules (proline and free amino acids) may appear the most striking feature for rice plants responding to induce water stress when having the $s u b 1 \mathrm{~A}$ locus. Now, the most independent and striking responses of $\operatorname{subl} A$, is variable in expression as a function of submergence through carbohydrate depletion. A temporary deferment of carbohydrate mobilization from source to sink and their rapid utilization that causes stagnation of growth has the similar magnitude under water stress also. Thereby, cv. Swarna Sub1 had significantly ( $\leq \leq 0.05)$ changed its carbohydrate content all through the stages of treatment, Still, under control a linear increase with the changes of $11.42 \%$ and $24.35 \%$ were recorded at 4 and 8 days as compared to initial day (Figure $3(\mathrm{~d})$ ). Therefore, the pattern of expression of Swarna Sub1 under submergence may also have some conformity with depleted water potential under drought condition. 


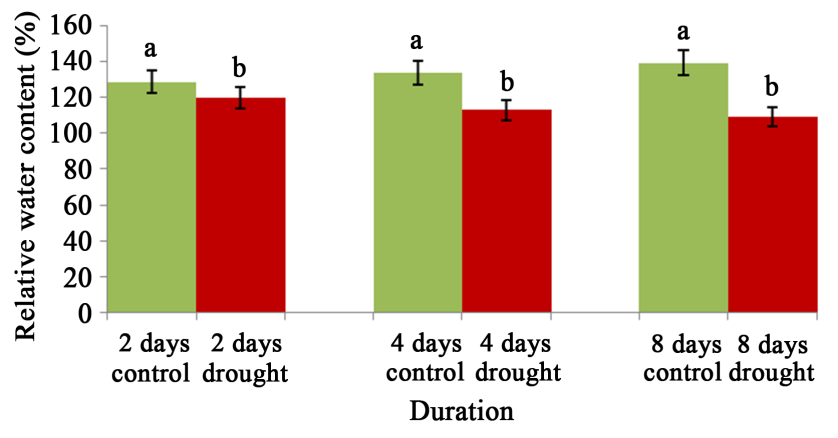

(a)

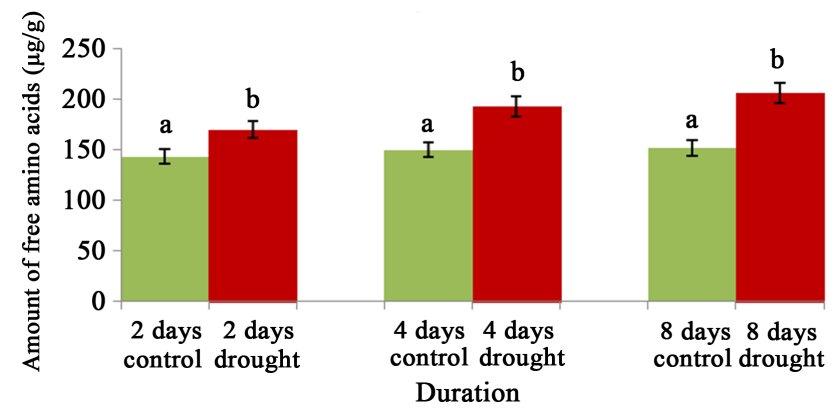

(c)

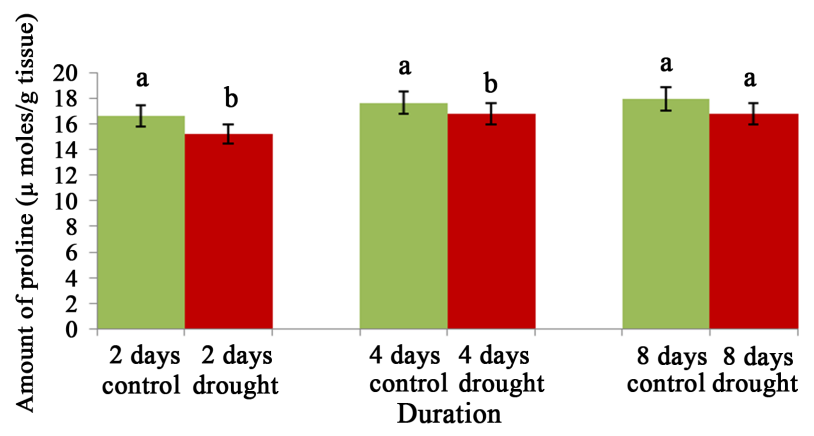

(b)

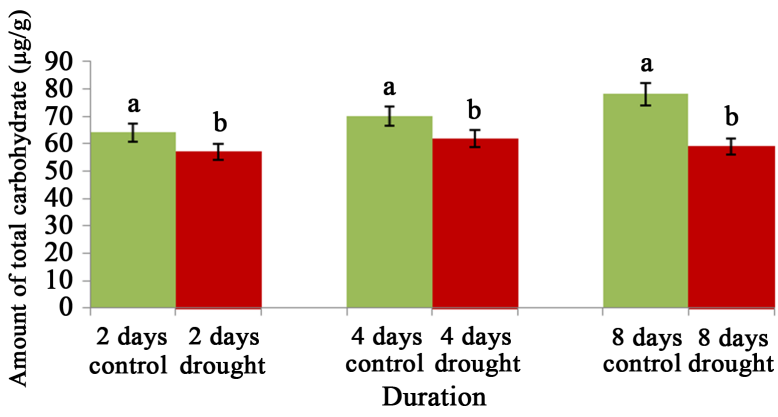

(d)

Figure 3. Changes of relative water content (a), proline (b), free amino acids (c) and total carbohydrates (d) content of cv. Swarna Sub1 through varying days under control and water stress. Data presented in bars with $\pm \mathrm{SE}(\mathrm{n}=3)$ from independent experimental sets at significance level $\mathrm{p} \leq 0.05$ (by student's $t$-test). Different letters on bar represent significant difference.

\subsection{Enzymatic Behaviour of Carbohydrate Metabolism of sub1A under Water Stress}

On the other hand, the solubilisation of carbohydrate is also attended with distinct behaviour under same condition of water stress. The activity of soluble invertase had the up regulation trend for the variety under control by maximum of 1.41 fold which had the value of $40.90 \%$ decline at 8 days of water stress treatment (Figure 4(a)). In a similar manner wall bound or apoplastic invertase which is one of the factors for elongation of internodes and leaf sheaths of land races bearing subl $A$ had the values of 1.69 fold increase and $71.42 \%$ decrease under control and water stress treatment respectively at 8 days (Figure 4(b)).

\section{Discussion}

It is quite discussed in plethora of citation that $s u b 1 A$ locus in rice is proficiently meant for quiescence strategies under submergence. Still, its validity needs more strengthening under condition of drying soil, the later may occasionally arises on delayed monsoon in low land rice culture [16]. From the results it appears that the $\operatorname{subl} A$ locus had its well responded reactions to ongoing duration of water deficit stress. Initially the dry matter accumulation through photosynthetic rate (NAR) significantly varied from 2 to 8 days duration of water stress. The stressed seedlings were undoubtedly deprived with fall in RGR than controlled ones. In this respect NAR could be substantiated with functional modules of total 


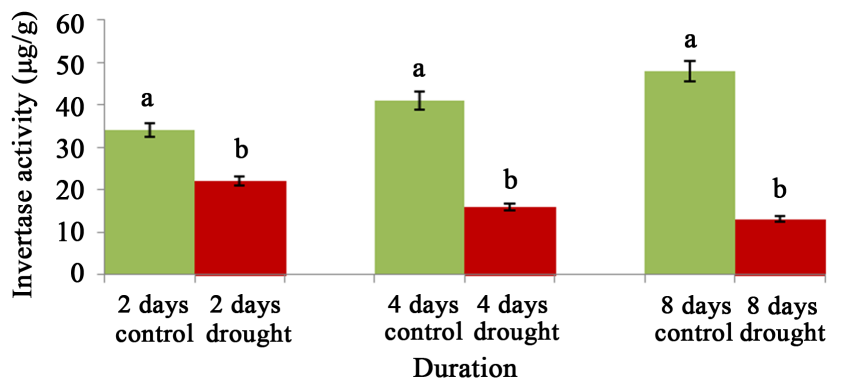

(a)

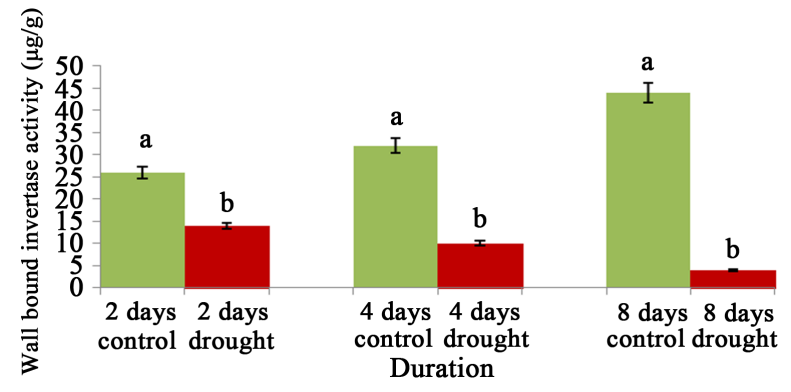

(b)

Figure 4. Changes of soluble invertase (a) and wall bound invertase (b) activity of cv. Swarna Sub1 through varying days under control and water stress. Data presented in bars with \pm SE $(n=3)$ from independent experimental sets at significance level $p \leq 0.05$ (by student's $t$-test). Different letters on bar represent significant difference.

leaf biomass. In rice, different ontogenic stages of growth are varied with the capacity of photosynthetic efficiencies by foliages except at the senescence period [17]. In seedling condition most of the photo-assimilate are partitioned into development of culm and release of tillers. This is required exclusively for growth and maintenance respiration for plant viability particularly, in seedling condition against water stress. This leads to deprivation of partitioning of biomass for dry matter accumulation mostly in the foliages and thus NAR through LMR is also compromised. Moreover, water deficit was vividly established with changes of RWC of the plants under 2, 4 and 8 days of water withholding. RWC is an indirect reflection of water status/hydration level of the tissue that has commonly been exercised in crop plants, more specifically for the initial screening [18]. Herein, cv. Swarna Sub1 had consistently varied with RWC, more towards longer duration as compared to control. RWC has a good correlation with mesophyll cell conductance that otherwise impact the NAR [19]. NAR, an accurate carbon acquisition over the photo-respiratory and respiratory loss is directly related to growth coordination through RGR and SLW. Thus, the rate of RGR was more depleted in case of $s u b 1 A$ under stress than control. This pattern of RGR may also be corroborated with LMR in the plants under water stress. The later being the exceptional characteristic features for cv. Swarna Sub1 that might not be bothering any changes for LMR contributing the values of SLW. In general, submergence is more observed to induce a linear growth of culm of seedlings with foliages [20]. This is typified in case of escape strategy when $\operatorname{subl} A$ may occasionally be active to over-express ethylene and total suppression of biomass, the results. Still, in the present case $\operatorname{subl} A$ also conferred to minimize the growth under water withholding. It also may predict the $\operatorname{subl} A$ allele would be confirmed for restriction of dry matter in a similar manner regardless of submergence and water stress. SLW which actualizes light absorption by the canopy and its conversion of photosynthetic dry matter was convincingly more consistent all through period of stress. Depletion of water status through poor root hydraulic conductivity under submergence of rice might also be linked with fall in water potential under water stress [21]. Regardless of rice cultivars roots appear more reliable vegetative organs to sensitize the excess or depletion of soil moisture 
tension. Swarna Sub1 had not been reported earlier with genetic elasticity for root behaviour under the control of quiescence mechanism. This has been expected under soil moisture deficit for regulated root growth as in present experiment. There had realised not any significant variation of root growth under water stress in comparison to control. Therefore, for the adaptability of root and its growth more towards higher water potential gradient in soil is failed when Swarna Sub1 is exposed to water stress. In rice, the water stress tolerance is characterized with a QTL that induces more linear growth of root than shoot. The roots consume more photosynthetic solutes to transport basipetally depriving the shoot growth [22]. One of the major mandates of $\operatorname{subl} A$ QTL in rice is to down regulate the carbon metabolism and its allocation to growing tissues resulting inhibited growth [23]. Still, this may not be applicable for the fate of $s u b 1 A$ QTL under water stress. The root growth is restricted and might be due to loss of viability or even degeneration of root tips under elevated oxidative status of water stress. Under drought, ethylene accumulation in apical zone of roots can induce earlier abscission following senescence in general [24]. It could also be quite expected $\operatorname{sub} 1 A$ element may have some negative feedback to over express the ethylene on the meristematic tissue of root under induced water stress.

With regards to growth performance it is quite obvious that $\operatorname{subl} A$ would be affected photosynthetically under water stress. Photosynthetic activities through coordination of pigments in land races of rice with $\operatorname{subl} A$ had hardly been studied. In the present experiment, we find a consistent fall in chlorophyll and lycopene content along with carotenoids through the duration of water stress. Besides, chlorophyll and lycopene, the carotenoids are regarded as accessory pigments for light harvesting mechanism as well as photo protective measures. Therefore, it is quite obvious that $\operatorname{subl} A$ allele could be prone to induce oxidative damages out of low concentration of carotenoids under water stress [25]. Through duration of stress $s u b 1 A$ experienced the level of nitrogen content in discriminating manner for proline and free amino acids. Swarna $s u b 1 A$ locus would be receptive to osmotic adjustment by lesser accumulation of proline under water stress. This may or may not have any compatibility in service of osmotic adjustment by normal proline expression. Therefore, insensitivity to proline as a cellular marker for water stress may not be adhered to $\operatorname{subl} A$. To advocate for compatible solutes under depleted soil moisture tension, plants have choice for the other bio-molecules like soluble sugars and free amino acids etc. The free amino acids are facing two probabilities as already reported under submergence in rice cultivars. Proline being hydrolysed product of proteins and also through its biosynthetic pathway with nitrogen assimilation is paid as osmoticum also. However, a significant increase of free amino acids over control but not through ongoing stress period may be an indication for hydrolysis of protein. In earlier reports, $s u b 1 A$ is characterized in a stable manner for regulation of the protein metabolism [26]. Carbohydrate metabolism appears to be more striking features in present experiment. The activity of invertase could also 
be anticipated for synthesis of compatible solutes (mostly soluble sugars) from hydrolysis of storage carbohydrate. The down regulation of invertase as compared to control signifies the failure of such development of compatible solutes for $\operatorname{subl} A$ and thus moisture stress is established. Therefore, the nature of $\operatorname{subl} A$ locus in rice land races may not be necessarily synergistic in manner when interacts with depleted soil moisture instead of water logging or submergence.

\section{Conclusion}

The resemblance of tolerance mechanism for this allele with submergence is ensured mostly with restriction of vegetative growth. If $s u b 1 A$ is granted to implement quiescence strategy through suppression of growth (may be for intermediate period of submergence) the same could be applicable for tissue dehydration. The later requires the regulation of vegetative growth whereby the water loss is minimised vis-a-vis maintenance of turgidity. On that aspect the compatible solutes like proline and free amino acids may be a choice for rice seedlings when it experiences tissue dehydration instead of flooding. So, apart from practice of quiescence strategy subl $A$ locus may be assured or even tried under depleted soil moisture tension for its viability following growth and development. Still, the other modalities in cellular responses (mostly the anti-oxidation cascades) to adjust the water deficit are yet to decipher with execution of $\operatorname{subl} A$ in rice land races.

\section{Acknowledgements}

The work is financially supported by DST-PURSE programme applicable to University of Kalyani. The central instrument facility of University of Kalyani is also acknowledged for partial assistant in execution of chemical analysis.

\section{Conflict of Interest}

The authors have no conflict of interest.

\section{References}

[1] Adak, M.K., Banerjee, S., Ghosh, N., Mandal, C. and Dey, N. (2015) Plants Response to Water in Excess: An Overview of Its Molecular Mechanism. Physiological Efficiency for Crop Improvement, 353.

[2] Whitmore, J.S. (2000) Hardy and Drought-Evasive Cereal Crops. In: Whitmore, J.S., Ed., Drought Management on Farmland, Springer, London, 79-97. https://doi.org/10.1007/978-94-015-9562-9_8

[3] Mackill, D.J., Ismail, A.M., Singh, U.S., Labios, R.V. and Paris, T.R. (2012) 6 Development and Rapid Adoption of Submergence-Tolerant (Sub1) Rice Varieties. Advances in Agronomy, 115, 299-352. https://doi.org/10.1016/B978-0-12-394276-0.00006-8

[4] Jackson, M.B. (2007) Ethylene-Promoted Elongation: An Adaptation to Submergence Stress. Annals of Botany, 101, 229-248. https://doi.org/10.1093/aob/mcm237

[5] Vandoorne, B., Beff, L., Lutts, S. and Javaux, M. (2012) Root Water Uptake Dynam- 
ics of Cichorium intybus var. Sativum under Water-Limited Conditions. Vadose Zone Journal, 11, 328-343. https://doi.org/10.2136/vzj2012.0005

[6] Murashige, T. and Skoog, F. (1962) A Revised Medium for Rapid Growth and Bio Assays with Tobacco Tissue Cultures. Physiologia Plantarum, 15, 473-497.

https://doi.org/10.1111/j.1399-3054.1962.tb08052.x

[7] Sarkar, R.K. and Bhattacharjee, B. (2011) Rice Genotypes with SUB1 QTL Differ in Submergence Tolerance, Elongation Ability during Submergence and Re-Generation Growth at Re-Emergence. Rice, 5, 7. https://doi.org/10.1007/s12284-011-9065-Z

[8] Withman, F.H., Blaydes, D.F. and Devlin, R.M. (1971) Experiments in Plant Physiology. Van Nostrand, New York, 245.

[9] Zakaria, M., Simpson, K., Brown, P.R. and Krstulovic, A. (1979) Use of Reversed-Phase High-Performance Liquid Chromatographic Analysis for the Determination of Provitamin A Carotenes in Tomatoes. Journal of Chromatography A, 176, 109-117. https://doi.org/10.1016/S0021-9673(00)92091-0

[10] Bates, L.S., Waldren, R.P. and Teare, I.D. (1973) Rapid Determination of Free Proline for Water-Stress Studies. Plant and Soil, 39, 205-207.

https://doi.org/10.1007/BF00018060

[11] Misra, P.S., Mertz, E.T. and Glover, D.V. (1975) Studies on Corn Proteins. VIII. Free Amino Acid Content of Opaque-2 Double Mutants. Cereal Chemistry, 52, 844-848.

[12] Barrs, H.D. and Weatherley, P.E. (1962) A Re-Examination of the Relative Turgidity Technique for Estimating Water Deficits in Leaves. Australian Journal of Biological Sciences, 15, 413-428. https://doi.org/10.1071/BI9620413

[13] Yoshida, S., Forno, D.A. and Cock, J.H. (1971) Laboratory Manual for Physiological Studies of Rice. Laboratory Manual for Physiological Studies of Rice, The International Rice Research Institute, Los Baños, Laguna.

[14] Bradford, M.M. (1976) A Rapid and Sensitive Method for the Quantitation of Microgram Quantities of Protein Utilizing the Principle of Protein-Dye Binding. Analytical Biochemistry, 72, 248-254. https://doi.org/10.1016/0003-2697(76)90527-3

[15] Adak, M.K., Ghosh, N., Dasgupta, D.K. and Gupta, S. (2011) Impeded Carbohydrate Metabolism in Rice Plants under Submergence Stress. Rice Science, 18, 116-126. https://doi.org/10.1016/S1672-6308(11)60017-6

[16] Datta, A., Ullah, H. and Ferdous, Z. (2017) Water Management in Rice. In: Chauhan, B.S., Jabran, K. and Mahajan, G., Eds., Rice Production Worldwide, Springer International Publishing, Berlin, 255-277.

https://doi.org/10.1007/978-3-319-47516-5_11

[17] Takai, T., Kondo, M., Yano, M. and Yamamoto, T. (2010) A Quantitative Trait Locus for Chlorophyll Content and Its Association with Leaf Photosynthesis in Rice. Rice, 3, 172-180. https://doi.org/10.1007/s12284-010-9047-6

[18] Abdallah, M.B., Methenni, K., Nouairi, I., Zarrouk, M. and Youssef, N.B. (2017) Drought Priming Improves Subsequent More Severe Drought in a Drought-Sensitive Cultivar of Olive cv. Chétoui. Scientia Horticulturae, 221, 43-52.

https://doi.org/10.1016/j.scienta.2017.04.021

[19] Kar, M., Mishra, B.K. and Sahu, A.K. (2008) 10 Adaptation of Rice Plants to Drought Prone Environments. Developments in Physiology, Biochemistry and Molecular Biology of Plants, 2, 269.

[20] Imaz, J.A., Giménez, D.O., Grimoldi, A.A. and Striker, G.G. (2013) The Effects of Submergence on Anatomical, Morphological and Biomass Allocation Responses of 
Tropical Grasses Chloris gayana and Panicum coloratum at Seedling Stage. Crop and Pasture Science, 63, 1145-1155. https://doi.org/10.1071/CP12335

[21] Mayoral, C., Pardos, M., Sánchez-González, M., Brendel, O. and Pita, P. (2016) Ecological Implications of Different Water Use Strategies in Three Coexisting Mediterranean Tree Species. Forest Ecology and Management, 382, 76-87. https://doi.org/10.1016/j.foreco.2016.10.002

[22] Sánchez-Calderón, L., Ibarra-Cortés, M.E. and Zepeda-Jazo, I. (2013) Root Development and Abiotic Stress Adaptation. In: Vahdati, K. and Leslie, C., Eds., Abiotic Stress-Plant Responses and Applications in Agriculture, InTech, London. https://doi.org/10.5772/55043

[23] Ramakrishnan, M., Ceasar, S.A., Vinod, K.K., Duraipandiyan, V., Krishna, T.A., Upadhyaya, H.D., Ignacimuthu, S., et al. (2017) Identification of Putative QTLs for Seedling Stage Phosphorus Starvation Response in Finger Millet (Eleusine coracana L. Gaertn.) by Association Mapping and Cross Species Synteny Analysis. PLoS ONE, 12, e0183261. https://doi.org/10.1371/journal.pone.0183261

[24] Savada, R.P., Ozga, J.A., Jayasinghege, C.P., Waduthanthri, K.D. and Reinecke, D.M. (2017) Heat Stress Differentially Modifies Ethylene Biosynthesis and Signaling in Pea Floral and Fruit Tissues. Plant Molecular Biology, 95, 313-331.

https://doi.org/10.1007/s11103-017-0653-1

[25] Simova-Stoilova, L., Vassileva, V. and Feller, U. (2016) Selection and Breeding of Suitable Crop Genotypes for Drought and Heat Periods in a Changing Climate: Which Morphological and Physiological Properties Should Be Considered? Agriculture, 6, 26. https://doi.org/10.3390/agriculture6020026

[26] Li, J., Wu, L., Foster, R. and Ruan, Y.L. (2017) Molecular Regulation of Sucrose Catabolism and Sugar Transport for Development, Defence and Phloem Function. Journal of Integrative Plant Biology, 59, 322-335. https://doi.org/10.1111/jipb.12539

\section{Abbreviations}

RGR, relative growth rate; LMR, leaf mass ratio; SLW, specific leaf weight; NAR, net assimilation rate; RWC, relative water content; QTL, quantitative trait loci. 